\title{
West Haven Hepatic Encephalopathy Grade 1
}

National Cancer Institute

\section{Source}

National Cancer Institute. West Haven Hepatic Encephalopathy Grade 1. NCI Thesaurus.

Code C117825.

Mild - symptoms or pathological characteristics include trivial lack of awareness, shortened attention span, impaired ability to perform addition or subtraction, hypersomnia, insomnia, or inversion of sleep pattern, euphoria, depression or irritability, mild confusion, slowing of ability to perform mental tasks. (Adapted from: Hepatic Encephalopathy. David C Wolf. http://emedicine.medscape.com/article/186101overview.) 